\title{
First physics with hadrons and the underlying event at CMS
}

\section{Ferenc Siklér* for the CMS Collaboration}

KFKI Research Institute for Particle and Nuclear Physics, Budapest, Hungary

E-mail: sikler@rmki.kfki.hu

The early data from LHC will allow the first look at minimum bias p-p collisions initially at the center-of-mass energies of 10 and later $14 \mathrm{TeV}$. The measurements of the basic observables will also serve as an important tool for calibrating and understanding the CMS detector at start-up.

We present the plans of the CMS collaboration to measure cross sections and differential yields of charged particles (unidentified or identified pions, kaons and protons) and neutrals $\left(\mathrm{K}_{\mathrm{S}}^{0}\right.$, lambdas and anti-lambdas) produced in inelastic p-p collisions at $14 \mathrm{TeV}$, as well as their multiplicity distributions. The tracking of charged particles is possible down to about $100 \mathrm{MeV} / \mathrm{c}$, with good efficiency and negligible fake rate. The yield of charged kaons and protons can be extracted for total momenta below 0.8 and $1.5 \mathrm{GeV} / \mathrm{c}$, respectively. Comparisons of the results to theoretical models are discussed. Studies of the underlying event at CMS are presented by examining charged particle and momentum densities in the transverse region in charged particle jet production. The possibility of discriminating among models is shown.

Physics at LHC 2008

29 September - 4 October, 2008

Split, Croatia

\footnotetext{
*Speaker.
} 

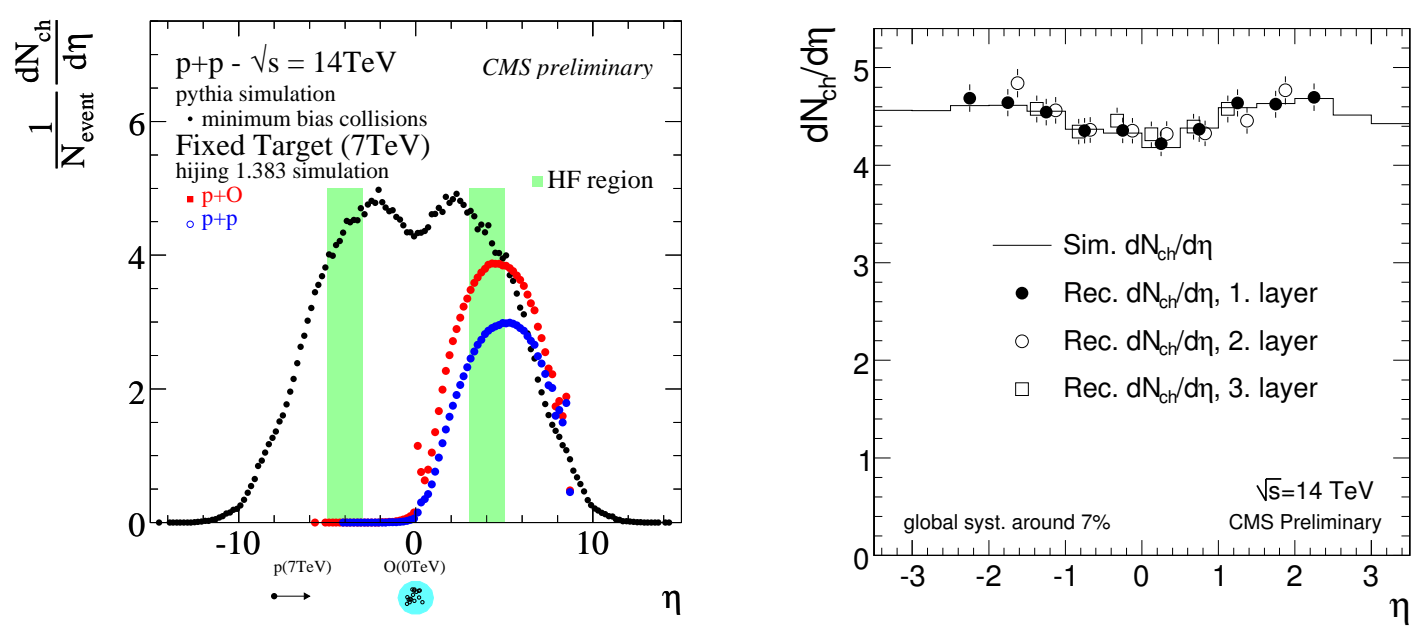

Figure 1: Left: The number of charged particles per unit pseudo-rapidity for minimum bias collisions compared to beam gas collisions occurring at the center of the detector. Right: Charged particle $d N_{c h} / d \eta$ distributions from generated (histogram) and reconstructed (symbols) p-p events at $14 \mathrm{TeV}$. Error bars show the statistical errors corresponding to 5000 events.

The CMS experiment at the LHC is a general purpose detector designed to explore physics at the TeV energy scale $[1,2]$. It has a large acceptance and hermetic coverage. The various subdetectors are: a silicon tracker with pixels and strips $(|\eta|<2.4)$; electromagnetic $(|\eta|<3)$ and hadronic $(|\eta|<5)$ calorimeters; and muon chambers $(|\eta|<2.4)$ [3]. The acceptance is further extended with forward detectors: CASTOR $(5.2<|\eta|<6.6)$ and Zero Degree Calorimeters $\left(\left|\eta_{\text {neutrals }}\right|>8.3\right)$. CMS detects leptons and both charged and neutral hadrons. This example analysis uses 2 million inelastic p-p collisions. They have been generated by the PYTHIA event generator [4].

In case of very low initial intensity the events can be taken by a special high level trigger, requiring at least one or two reconstructed tracklets in the pixel detector. This trigger has very low bias and an efficiency of about $88 \%$ for inelastic p-p collisions. Another type of trigger will be based on counting towers, with energy above the detector noise level, in both forward hadronic calorimeters (HF, $3<|\eta|<5$ ). A minimal number of hits (1, 2 or 3) will be required on one or on both sides, an energy threshold value of $1.4 \mathrm{GeV}$ is used in the hit definition [5]. The efficiency of this one-sided trigger for inelastic p-p collisions is about $89 \%$. The double-sided trigger is less efficient (about 59\%), but it is also less sensitive to beam-gas background (Fig. 1-left). Once the luminosity is high enough, events can also be taken with the so called zero-bias trigger based on a random clock each bunch crossing.

Charged hadrons with $p_{T}$ larger than $30 \mathrm{MeV} / c$ can leave hits in the layers of the pixel detector. Its fine segmentation and small occupancies allow for the measurement of the $\eta$ distribution of charged hadrons by counting the number of reconstructed hits [6]. With help of the length of the pixel hit cluster in beam direction, the position of the interaction vertex can be estimated. It also helps to remove background hits at higher $\eta$ if their size is incompatible with the found vertex position. The number of detected hits has to be corrected for non-primary origin: looping particles, secondaries, decay products. A systematic error of 7\% is expected (Fig. 1-right). The method is attractive since it does not need particle tracking and it is insensitive to the alignment of the tracker. 

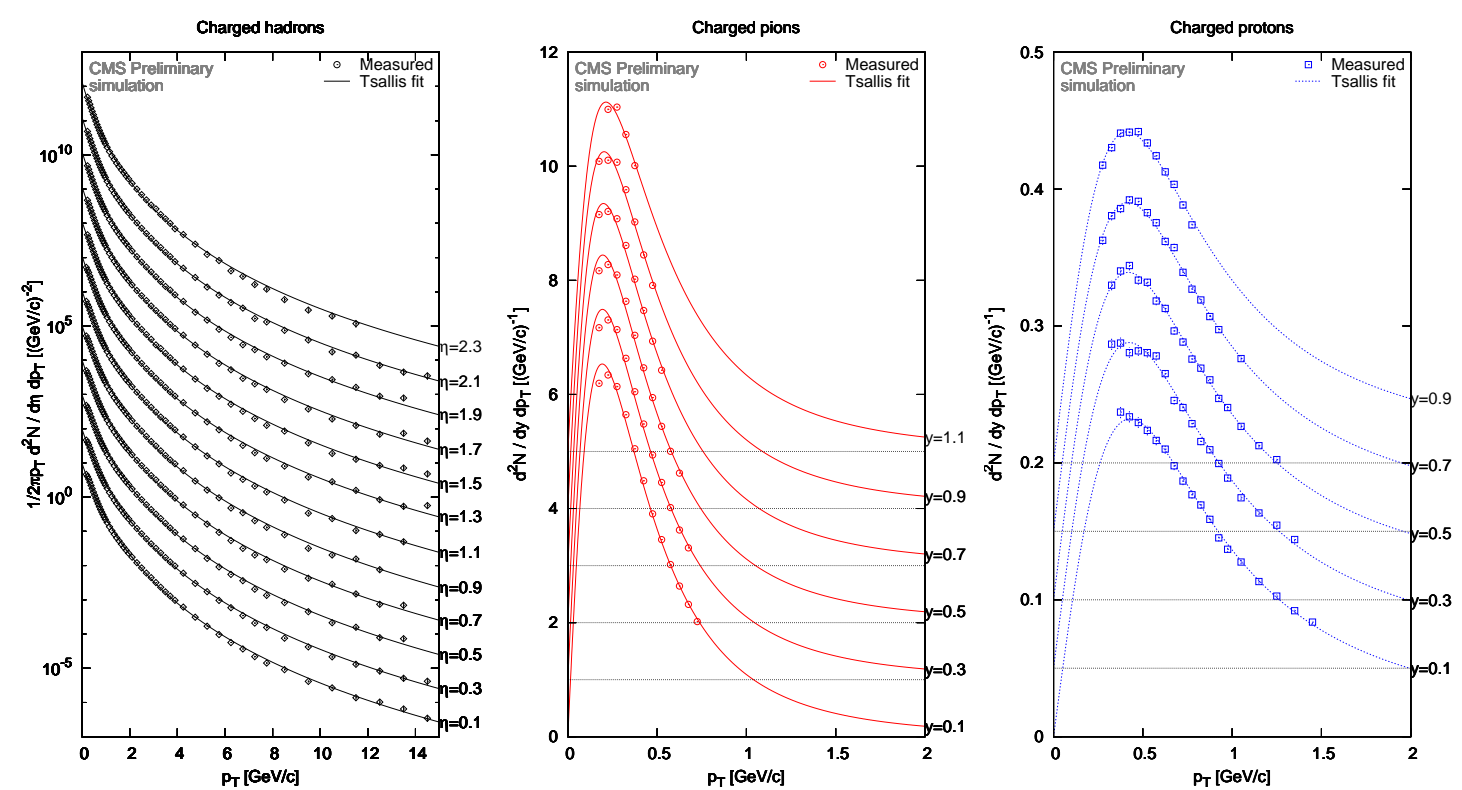

Figure 2: Selection of particle spectra. Left: Measured invariant yields of charged hadrons in the range $0<|\eta|<2.4$. Right: Measured differential yields of identified charged pions and protons in the range $0<|y|<1.2$. Measured values and empirical fit functions are plotted, with a series of 0.2 unit wide bins. Values are successively multiplied by 10 or shifted for clarity.

Both pixel and strip silicon tracker detectors are used for the reconstruction of charged particles. With a modified hit triplet finding algorithm the pixel detector can be employed for the reconstruction of low $p_{T}$ charged particles $[2,8,9]$. The acceptance of the method extends down to $0.1,0.2$ and $0.3 \mathrm{GeV} / c$ in $p_{T}$ for pions, kaons and protons, respectively. The obtained pixel tracks are used for finding and fitting the primary vertex or vertices [7]. The found vertices are reused, ensuring that the track comes from an interaction point. This brings the fake track rate down to per mille levels. The measured shape of tracker hits is compared to the dimensions predicted from the local direction of the trajectory. This filter helps to eliminate incompatible trajectory candidates at an early stage. At the end, the trajectory is refitted with the primary vertex constraint.

The hadron spectra are corrected for particles of non-primary origin. Their main source is the feed-down from weakly decaying resonances. While the correction is around $2 \%$ for pions, it can go up to $15 \%$ for protons with $p_{T} \approx 0.3 \mathrm{GeV} / c$. The resonances $\mathrm{K}_{\mathrm{S}}^{0}, \Lambda$ and $\bar{\Lambda}$ can be extracted from the measured data.

Charged particles can be singly identified or their yields can be extracted (identification in the statistical sense) using deposited energy in the pixel and strip silicon tracker, with help of the truncated mean estimator [9]. The distribution of $\log \mathrm{d} E / \mathrm{d} x$ can be successfully fitted in slices of momentum. The fit function is a sum of properly scaled Gaussians for each particle species: here pions, kaons and protons are assumed. The relative resolution of $\mathrm{d} E / \mathrm{d} x$ for tracks with average number of hits $(\sim 15)$ is around 5-7\%. The yield of kaons can be extracted if $p<0.8 \mathrm{GeV} / c$ and that of protons if $p<1.5 \mathrm{GeV} / c$. Both limits correspond to approximately $3 \sigma$ separation.

The measured invariant yields of charged hadrons are shown in Fig. 2-left, as a function of $p_{T}$ and in narrow $\eta$ bins. (Results refer to the sum of positively and negatively charged particles. 

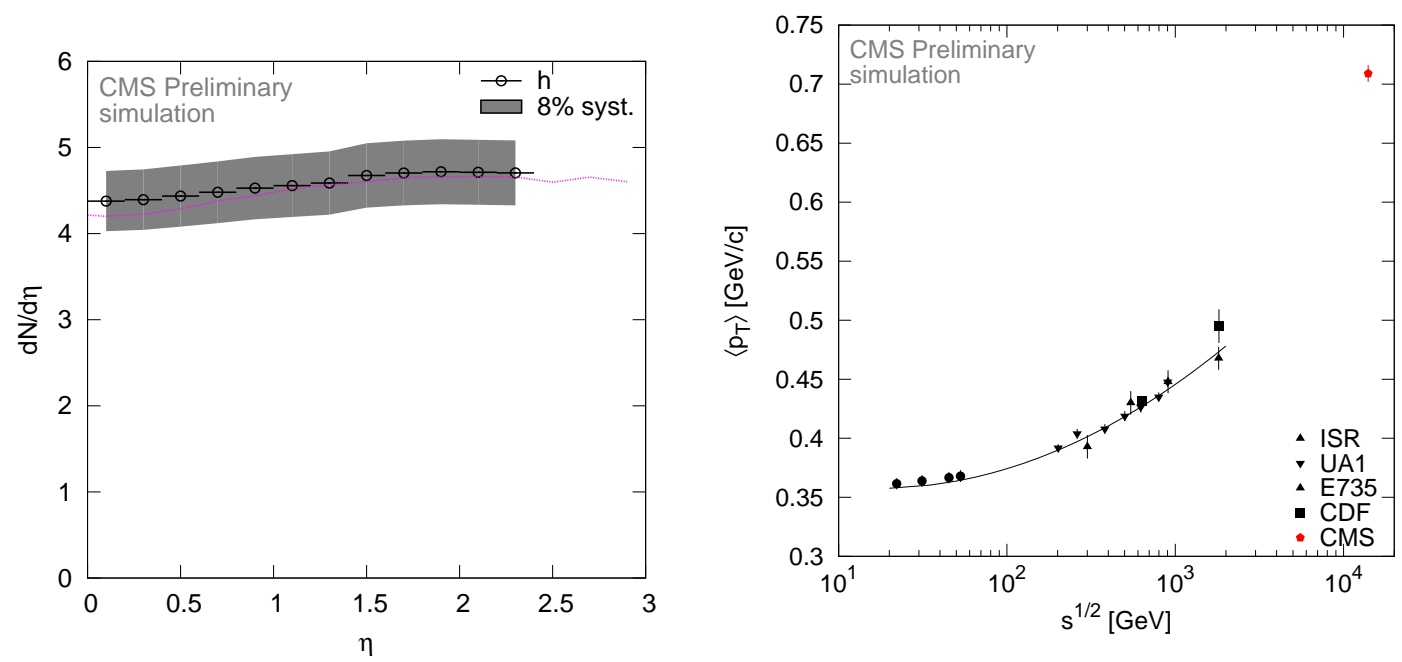

Figure 3: Left: Pseudorapidity density distribution of charged hadrons. The distribution from simulated tracks is given by the purple curve for comparison. Right: Energy dependence of average transverse momentum of unidentified charged hadrons at $\eta \approx 0$. The curve shows a quadratic fit on data points of other experiments [11]. The result of this analysis is shown with red pentagons.

Symmetric positive and negative $\eta$ bins are also added.) Measured differential yields of identified charged pions and protons are shown in Fig. 2-right. The obtained invariant yields were fitted by the Tsallis function [10], a function that successfully combines and describes both the low $p_{T}$ exponential and the high $p_{T}$ power-law behaviors. The pseudorapidity distribution of charged hadrons is shown in Fig. 3-left. The energy dependence of some measured quantities can also be studied (Fig. 3-right).

The study of the underlying event deals with all the activities of a single p-p interaction on top of the interesting hard process. Hence it covers several mechanisms: initial and final state radiation, the spectators, and multiple parton interactions, which is of particular interest today. Its activity is expected to grow with the energy scale of the main interaction. While there are many generator tunes describing data at Tevatron energies, they have several differences when extrapolating to LHC energy. The analysis looks at the topology of a p-p collision from the point of the leading charged jet [12]. (This latter defines a reference direction in the $\phi$ plane.) The region transverse to the jet is expected to be particularly sensitive to the characteristics of the underling event. The observables are the charged density $d N / d \phi d \eta$ and the energy density $d p_{T}^{\text {sum }} / d \phi d \eta$. The charged particles are reconstructed by standard methods with the CMS tracker [7]. The comparison of simulated and reconstructed quantities show a good discrimination power as function of $p_{T}^{\text {Chg jet }}$ (Fig. 4), for a given lower $p_{T}$ cut. Ratios of these observables with a cut of $0.9 \mathrm{GeV} / c$ and $1.5 \mathrm{GeV} / c$ are not sensitive to corrections since these are already absorbed in the ratio.

In summary, spectra and yields of charged and neutral particles (unidentified and identified) produced in inelastic proton-proton collisions can be measured with good precision with the CMS tracker. The study of the underlying event helps to tune energy dependence models, understand multiple parton interactions and to improve the QCD understanding of p-p collisions.

The author wishes to thank the Hungarian Scientific Research Fund and the National Office for Research and Technology (K 48898, H07-B 74296). 

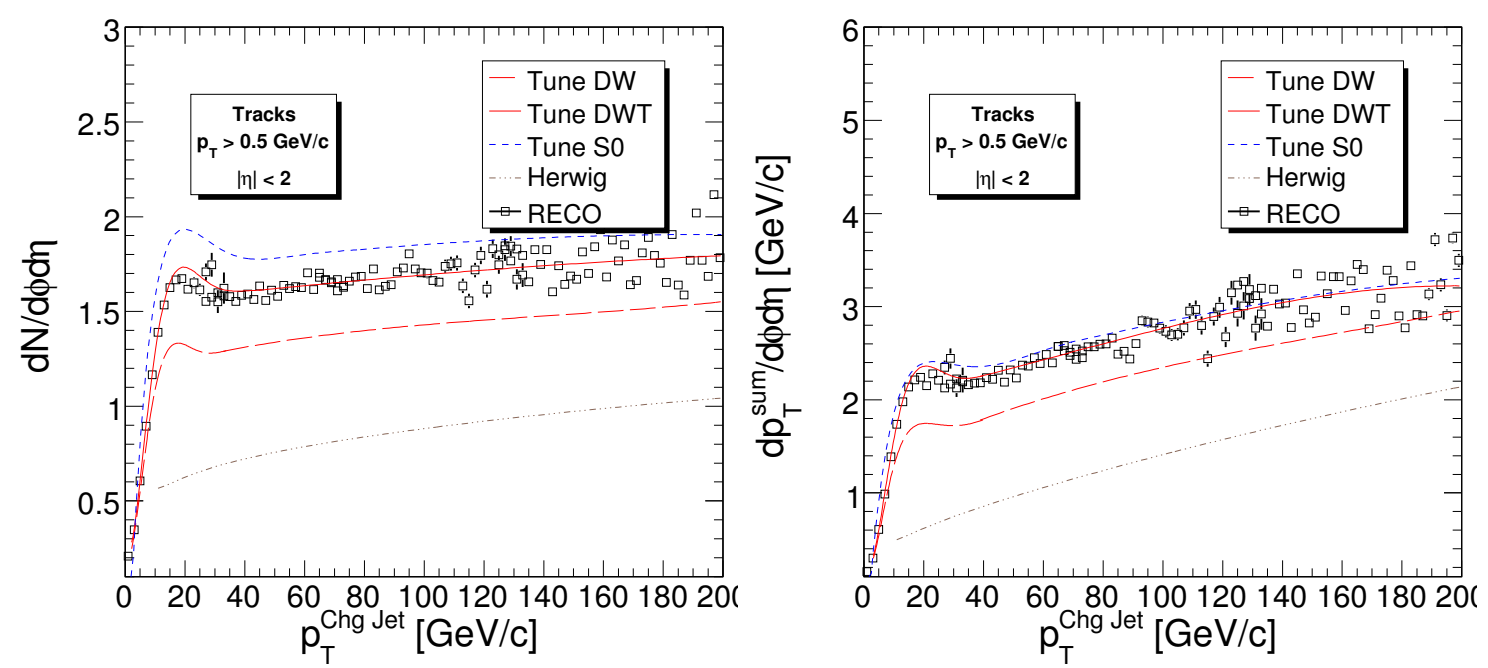

Figure 4: Densities $d N / d \eta d \phi$ (left) and $d p_{T}^{\text {sum }} / d \eta d \phi$ (right) for tracks with $p_{T}>0.9 \mathrm{GeV} / c$, as a function of the leading charged jet $p_{T}$, in the transverse region, for an integrated luminosity of $100 \mathrm{pb}^{-1}$.

\section{References}

[1] G. L. Bayatian et al. [CMS Collaboration], CMS technical design report, volume II: Physics performance, J. Phys. G 34 (2007) 995.

[2] D. d'Enterria et al., CMS physics technical design report: Addendum on high density $Q C D$ with heavy ions, J. Phys. G 34 (2007) 2307.

[3] R. Adolphi et al. [CMS Collaboration], The CMS experiment at the CERN LHC, JINST 3 (2008) S08004.

[4] T. Sjostrand, S. Mrenna and P. Skands, PYTHIA 6.4 physics and manual, JHEP 0605 (2006) 026.

[5] The CMS Collaboration, Zero bias and HF-based minimum-bias triggering for pp collisions at 14 TeV in CMS, CMS PAS QCD-07-002.

[6] The CMS Collaboration, Pseudorapidity distributions of charged hadrons in minimum bias p-p collisions at $\sqrt{\mathrm{s}}=14 \mathrm{TeV}$, CMS PAS QCD-08-004.

[7] S. Cucciarelli, M. Konecki, D. Kotliński and T. Todorov, Track reconstruction, primary vertex finding and seed generation with the Pixel Detector, CMS Note 2006/026. W. Adam, B. Mangano, T. Speer, T. Todorov, Track Reconstruction in the CMS tracker, CMS Note 2006/041. G. L. Bayatian et al. [CMS Collaboration], CMS physics: Technical design report, CMS-TDR-008-1.

[8] F. Siklér, Low p(T) hadronic physics with CMS, Int. J. Mod. Phys. E 16 (2007) 1819. F. Siklér [CMS Collaboration], Soft probes of high density QCD physics with CMS, J. Phys. G 35 (2008) 044045.

[9] The CMS Collaboration, Measurement of charged hadron spectra in proton-proton collisions at $\sqrt{s}=$ $14 \mathrm{TeV}, \mathrm{CMS}$ PAS QCD-07-001.

[10] C. Tsallis, Possible Generalization of Boltzmann-Gibbs Statistics, J. Statist. Phys. 52 (1988) 479.

[11] T. S. Ullrich, Experimental summary on global observables, hadron spectra and ratios, Nucl. Phys. A 715 (2003) 399.

[12] The CMS Collaboration, Measurement of the Underlying Event in Jet Topologies using Charged Particle and Momentum Densities, CMS PAS QCD-07-003. 